\title{
DETERMINACIÓN DE LOS NIVELES DE ACIDO LINOLEICO CONJUGADO (ALC) EN ALIMENTOS LÁCTEOS EN CHILE
}

\section{AMOUNTS OF CONJUGATE LINOLEIC ACID (ALC) IN LACTEAL FOODS IN CHILE}

\author{
Juan Pablo Avilez R. (1), Carolina Isabel Vilches S. (1), Marcelo Wladimir Alonzo V. (2)
}

(1) Facultad de Recursos Naturales. Universidad Católica de Temuco. Temuco. Chile

(2) Nestlé-Chile. Departamento de Nutrición. Francisco del Campo S/N. Fábrica Osorno - Planta Nestlé.

\begin{abstract}
The amounts of conjugated linoleic acid (ALC) - Isomers: cis-9, trans-11, trans-10, cis-12 and cis-10, cis-12-were measured in a milk tank of the NESTLE Company and of diary food in Chile and were analyzed through gas chromatography. The ALC was found higher $(p<0.05)$ in samples taken in the city of Osorno compared to milk of the city of Los Angeles. The highest value was found during spring in Osorno (1,72 g/100 g), while the lowest value $(0,415 \mathrm{~g} / 100 \mathrm{~g})$ was found in winter in Los Angeles. The average amount of ALC in milk powder and condensed milk was of 1,967 and 1,493 g/100 g, respectively, during 2004. Butter, cheese and cream had respectively an average of 1,502, 0,883 and 1,900 $\mathrm{g} / 100 \mathrm{~g}$. In conclusion, the diary products analyzed have a high value of ALC in Chile.
\end{abstract}

Key words: CLA; conjugated linoleic acid; milk; dairy products.

Este trabajo fue recibido el 18 de Julio de 2008 y aceptado para ser publicado el 20 de Marzo de 2009.

\section{INTRODUCCIÓN}

El ácido linoleico conjugado (ALC) se ha reportado como el ácido graso más benéfico para la salud humana (1). De los 20 a 28 posibles isómeros posicionales y geométricos del ALC solo cis- 9, trans-11 y trans-10, cis-12 han sido reportados con actividad biológica en el ser humano (2). El isómero cis- 9, trans-11 (C18:2) ó ácido octadecadienóico, es el producto intermedio de la biohidrogenación ruminal del ácido linoleico por acción de la bacteria Butyrivibrio fibrisolvens). Este isómero también puede ser sintetizado en los tejidos de la glándula mamaria por la enzima $\Delta-9$ desaturasa a partir de ácido vaccénico (TVA- trans-11 C18:1), otro intermediario en la biohidrogenación ruminal del ácido linoleico y $\alpha$ - linolénico (cis-9, cis-12, cis-15 C18:3) (3).

La $\Delta-9$ desaturasa también se encuentra en el intestino delgado y tejido adiposo de los rumiantes y humanos (4), sugiriendo que podría ocurrir síntesis endógena en estos tejidos. El isómero cis- 9 , trans-11 es considerado desde el punto de vista biológico como el más importante por sus efectos anticancerígenos en modelos de tejido en ratas y en humanos (5). El isómero cis- 9, trans-11 estaría relacionado además, a la disminución del tejido graso y la reducción del peso en las personas (6).

Otros beneficios del ALC serían la protección contra la arteriosclerosis (7), la caquexia y podría ser usado para tratar la diabetes (8).

Se reporta también un efecto estimulador del sistema inmune (9) y efectos antioxidantes (10). El ALC se ha encontrado en diversos alimentos como los lácteos, carnes, productos vegetales y alimentos de origen marino, siendo más alto en la leche y en la carne proveniente de animales rumiantes (11). Un 0,11 g/100g de ALC en grasa se ha reportado en la carne de conejos, un 1,20 $\mathrm{g} / 100 \mathrm{~g}$ en cordero y un $0.09 \mathrm{~g} / 100 \mathrm{~g}$ en carpa. En aceites vegetales y en margarinas corresponde a menos del 0.01 $\mathrm{g} / 100 \mathrm{~g}$ (12). En productos lácteos se ha encontrado entre un 0,40 - 1,7 g/100g de ALC del total de grasa en queso gauda, jurassic y Old Emmentales (13).

La cantidad de ALC en lácteos de origen bovina estaría afectada por muchos factores como son: la raza, días de lactancia del animal, tipo alimento utilizado en la alimentación, estacionalidad del año, entre otros; siendo la dieta el factor que tiene más efecto sobre el ALC de le- 
che fresca y en los productos lácteos (14). En este sentido la alimentación de vacas teniendo como base la pradera aumenta la cantidad de ALC (15) lo cual es importante en leche fresca y en la leche que será procesada. En el caso chileno se ha estudiado los ácidos grasos en leche bovina recepcionada en planta y su relación con la estacionalidad anual y el sector geográfico, determinándose niveles de ALC para la IX región de 1,75 g/100g y para la VIII región de 1,41 g/100g (16); sin embargo solo se menciona al isómero cis- 9 , trans-11 y no se tiene información de la cantidad de ALC en productos derivados de leche (queso, mantequilla, leche condensada y leche en polvo). Es por ello que este estudio tiene como objetivo determinar la cantidad de ALC y de sus isómeros, tanto de la leche recepcionada en planta como de productos lácteos derivados para consumo humano.

\section{MATERIAL Y MÉTODOS}

Muestras de silo: Se tomaron un total de 18 muestras de leche de silos (estanque) de recepción en la planta NESTLÉ-Chile ubicada en Osorno $(n=9)$ y en Los Ángeles (n=9) el año 2004 en tres estaciones del año: 3 en verano, 3 en otoño y 3 en primavera según protocolo (17).

Bases de productos: Se analizaron bases de productos lácteos procedentes de la NESTLÉ Llanquihue, Región de Los Lagos. Las bases de productos corresponden al proceso de extracción de la grasa de la leche que será ocupada en la elaboración de productos lácteos. Para ello se tomó muestras $(n=4)$ correspondientes a leche al $1 \%$ de materia grasa, al $2 \%$ de materia grasa, leche descremada y crema.

Productos lácteos: Para la leche condensada se tomaron muestras durante el año 2003, 2004 y 2005 $(n=48)$ de la planta Los Ángeles. Para la leche el polvo se tomaron muestras durante el año 2003, 2004 y 2005 $(n=48)$ de la planta Osorno. Para la leche líquida se tomaron muestras durante el año $2005(\mathrm{n}=10)$ de la planta Osorno. Además se muestreó mantequillas y quesos elaborados en Chile ( $\mathrm{n}=7$ cada uno).

Análisis de ALC en leche y de los isómeros: Se determinó la cantidad de ALC de los isómeros: cis-9, trans-11; trans-10, cis-12; y el isómero cis-10, cis-12 en el laboratorio de Acuicultura de la Universidad Católica de Temuco "Analacu". Se obtuvo un volumen de $100 \mathrm{ml}$ de leche provenientes de cada muestra ó $1 \mathrm{~g}$ de muestra según correspondía a un líquido o a un sólido. Para este análisis se procedió a la extracción de los lípidos, a partir de $100 \mathrm{mg}$ de muestra, de acuerdo a los procedimientos descritos por Folch (18). Este método consiste en homogeneizar la muestra en una mezcla de cloroformo/metanol $(2: 1, \mathrm{v} / \mathrm{v})$, y luego centrifugar para separar la fase hidrosoluble y orgánica. La fase orgánica queda retenida en el cloroformo y la hidrosoluble en el metanol, separándose la fase orgánica.

Una vez pesados los extractos lipídicos disueltos en el cloroformo, son guardados a $-80^{\circ} \mathrm{C}$ hasta la preparación para el análisis de los ácidos grasos. La metilación de los ácidos grasos de las muestras se realizó según la metodología recomendada por Morrison y Smith (19). A través del análisis cromatográfico se determinó el perfil de ácidos grasos como ésteres metílicos, en un cromatógrafo Gas- Líquido modelo HP 6890 (Hewlett Packard), Detector de Ionización de Llama (FID), una columna capilar SP-2380 (30 m, 0,25 mm i.d. con 0,20 $\mu \mathrm{m}$ de espesor de fase estacionaria; Supelco Inc., Bellefonte, PA) gas de arrastre He. Se usaron estándares Supelco Inc 37 para estimar los factores de repuesta (RF) de los ácidos grasos y su posterior cuantificación mediante el software HPCHEM Stations, siendo expresados como porcentaje, de acuerdo al total de los ácidos grasos identificados.

Análisis estadísticos: Se realizó estadística descriptiva, media y desviación estándar y comparación múltiple DMS (Silos de leche), utilizando el Programa Statistical Package for the Social Sciences SPSS versión 13,0 para Windows (SPSS Inc, Chicago IL, USA).

\section{RESULTADOS}

\section{Niveles de ALC en productos lácteos}

En la tabla 1 se observa que la cantidad de ALC por estación del año y por región geográfica presenta diferencias significativamente $(\mathrm{p}<0,05)$ encontrándose mayores niveles de ALC en los silos de primavera y localizados en Osorno; sin embargo, no se encontraron diferencias entre la estación de otoño entre regiones. Los productos lácteos presentaron niveles promedios muy variados en cuanto a sus contenidos de ALC, que va de $0,883 \mathrm{~g} / 100$ $\mathrm{g}$ de grasa en queso, a 1,967 g/100 $\mathrm{g}$ en leche en polvo y a $1,502 \mathrm{~g} / 100 \mathrm{~g}$ en mantequilla. En leche condensada se encontró niveles de 0,960 a 2,200 de ALC. También se observa variación en los años analizados en la leche condensada y en leche el polvo, encontrándose mayores niveles de ALC en al año 2004, con respecto al 2003 y 2005. Por otro lado las bases de productos también son altas en ALC, sobre todo en cremas, debido a la concentración alta de grasa en estos productos.

\section{Isómeros del ALC en productos lácteos}

La tabla 2 muestra los isómeros de ALC. Podemos observar que el isómero cis-9, trans-11 se encuentra en un rango que va de 0,262 para el silo de leche en verano en los Ángeles y un máximo de 1,260 en mantequilla de la región de los Lagos. Este isómero porcentualmente 
representa en promedio el 53\% del total de ALC siendo más alto en la región de los Lagos. En el caso de la leche condensada este isómero varía de 0.437-0.738 $\mathrm{g} / 100 \mathrm{~g}$ dependiendo el año estudiado. El isómero cis-9, trans-11 en el queso y mantequilla presentaron un rango de $0,370-1,470 \mathrm{~g} / 100 \mathrm{~g}$ y $1,220-2,220 \mathrm{~g} / 100$ g respectivamente.

El isómero trans-10, cis-12 tiene un rango de 0,152 a 0,430 en los silos de leche, y no se encontró en las muestras de otoño y primavera.

El isómero cis-10, cis-12 lo encontramos en alta proporción en silo de leche en primavera en la planta de Osorno y también se encontró en alta concentración en leche condensada y en leche en polvo. En relación a la cantidad total de ALC alcanzó sobre un $50 \%$ en leche condensada, crema y leche en polvo; siendo el mayor isómero encontrado en Osorno en los silos de primavera. Este estudio determinó que el isómero cis10 , cis-12 se presenta en alta cantidad seguido del cis-9, trans-11, por lo cual cobra gran importancia determinar su rol biológico.

\section{DISCUSIÓN}

\section{Niveles de ALC en productos lácteos}

A nivel internacional se han reportado valores máximos de 1,16 g/100g de ALC (12) en Alemania, de $0,04 \mathrm{~g} / 100 \mathrm{~g}$ de ALC en Francia (20) y en Portugal 0,72 $\mathrm{g} / 100 \mathrm{~g}$ (21) en grasa de leche natural. Estos valores de ALC son más bajos que los encontrados en grasa láctea en la Región del Bio Bio que fue de $1,41 \mathrm{~g} / 100 \mathrm{~g}$ y más bajo aún que en la Región de los Lagos que fue de $1,73 \mathrm{~g} / 100 \mathrm{~g}$. También corresponde a valores más

\section{TABLA 1}

ALC total en la grasa de leche en silos y en productos lácteos.

\begin{tabular}{|c|c|c|c|}
\hline \multirow[t]{2}{*}{ Variable } & \multicolumn{3}{|c|}{ ALC Total ${ }^{1}(\mathrm{~g} / 100 \mathrm{~g}$ ácidos grasos) } \\
\hline & $\mathbf{x} \pm \mathbf{D E}^{(1)}$ & mínimo & máximo \\
\hline \multicolumn{4}{|l|}{ Silo de leche } \\
\hline Verano Los Ángeles & $0,415 \pm 0,119 \mathrm{f}^{(2)}$ & 0,278 & 0,494 \\
\hline Otoño Los Ángeles & $0,823 \pm 0,085 \mathrm{~cd}$ & 0,740 & 0,910 \\
\hline Primavera Los Ángeles & $0,930 \pm 0,283 b$ & 0,415 & 1,330 \\
\hline Verano Osorno & $0,608 \pm 0,014 \mathrm{e}$ & 0,595 & 0,623 \\
\hline Otoño Osorno & $0,887 \pm 0,137 \mathrm{c}$ & 0,740 & 1,010 \\
\hline Primavera Osorno & $1,720 \pm 1,207 \mathrm{a}$ & 0,550 & 2,960 \\
\hline \multicolumn{4}{|l|}{ Bases de productos (\%MG) } \\
\hline Leche al $1 \%$ & 0,620 & & \\
\hline Leche al $2 \%$ & 0,599 & & \\
\hline Leche descremada & $1,350 \pm 0,626$ & 0,630 & 1,770 \\
\hline Crema & $1,900 \pm 0,056$ & 1,780 & 1,948 \\
\hline \multicolumn{4}{|l|}{ Productos lácteos } \\
\hline Mantequilla & $1,502 \pm 0,409$ & 1,260 & 2,220 \\
\hline Quesos & $0,883 \pm 0,555$ & 0,370 & 1,470 \\
\hline Leche condensada 2003 & $1,033 \pm 0,237$ & 0,730 & 1,460 \\
\hline Leche condensada 2004 & $1,493 \pm 0,491$ & 1,140 & 2,200 \\
\hline Leche condensada 2005 & $1,312 \pm 0,260$ & 0,960 & 1,610 \\
\hline Leche en polvo 2003 & $1,430 \pm 0,258$ & 1,060 & 2,140 \\
\hline Leche en polvo 2004 & $1,967 \pm 0,369$ & 1,610 & 2,590 \\
\hline Leche en polvo 2005 & $1,463 \pm 0,211$ & 1,240 & 1,790 \\
\hline
\end{tabular}


bajos encontrados en época de primavera-verano $(1,75$ $\mathrm{g} / 100 \mathrm{~g}$ ) y a valores inferiores que se presentaron en la época de otoño-invierno $1,59 \mathrm{~g} / 100 \mathrm{~g}$; sin embrago, se señala al cis-9, trans-11 como único isómero encontrado (16), en el trabajo Francés se menciona al ALC total (20) y para el caso de Portugal se dan a conocer 17 isómeros de ALC, señalándose que el cis-9, trans-11 esta en alta proporción. En nuestro estudio el total de ALC se atribuyó a tres isómeros detectados principalmente por el tipo de columna utilizada. La cantidad de ALC encontrada estaría relacionada con los sistemas de producción diferencial en cada región y principalmente por el tipo de alimentación utilizada en los animales. Para el caso de la región de Los Lagos, la alimentación tiene como base la pradera (22) a diferencia de los sistemas productivos de la región del Bio-Bio donde los sistemas productivos de bovinos lecheros son principalmente estabulados. Por otro lado las variaciones estacionales del contenido de ALC en grasa láctea tienen directa relación con el crecimiento y la calidad de la pradera (15). El ALC aumenta de 2 a 3 veces en grasa láctea durante la primavera cuando las vacas son alimentadas a pastoreo y las concentraciones de ALC promediaron valores de $1,2 \mathrm{~g} / 100 \mathrm{~g}$ en esta estación, disminuyendo a $0,9 \mathrm{~g} / 100 \mathrm{~g}$ en verano e incrementándose nuevamente a $1,0 \mathrm{~g} / 100 \mathrm{~g}$ en otoño (23). Vacas que reciben toda su dieta desde pastoreo, producen grasa láctea con mayores contenidos de ALC comparados con vacas que consumen uno o dos tercios de su dieta en forma de pastoreo (24), por lo que incrementando los niveles de forraje fresco (pradera o forraje fresco cortado) se incrementan los niveles de TVA, ALC, y C18:3 (4). Esto cobra gran importancia

\section{TABLA 2}

Isómeros de ALC en la grasa de leche en silos y en productos lácteos.

Variable

$$
\begin{gathered}
\text { Cis-9, trans-11 } \\
\mathrm{x} \pm \mathrm{DE}^{(\mathbf{1})}
\end{gathered}
$$

\section{Isómeros (g/100 g ácidos grasos)}

$$
\begin{gathered}
\text { trans-10, cis-12 } \\
\mathrm{x} \pm \mathrm{DE}
\end{gathered}
$$

\section{Silo de leche}

Verano Los Ángeles

Otoño Los Ángeles

Primavera Los Ángeles

Verano Osorno

Otoño Osorno

Primavera Osorno

\section{Bases de productos (\% MG)}

Leche al $1 \%$

Leche al $2 \%$

Leche descremada

Crema

\section{Productos lácteos}

Mantequilla

Quesos

Leche condensada 2003

Leche condensada 2004

Leche condensada 2005

Leche en polvo 2003

Leche en polvo 2004

Leche en polvo 2005

$0,262 \pm 0,062$
$0,317 \pm 0,150$
$0,493 \pm 0,023$
$0,397 \pm 0,010$
$0,480 \pm 0,036$
$0,813 \pm 0,438$

0,389

0,385

$0,476 \pm 0,235$

$0,860 \pm 0,446$

$0,920 \pm 0,383$

$0,470 \pm 0,343$

$0,437 \pm 0,150$

$0,738 \pm 0,227$

$0,787 \pm 0,547$

$0,681 \pm 0,114$

$0,970 \pm 0,234$

$0,729 \pm 0,129$

$$
\begin{gathered}
0,152 \pm 0,057 \\
0,330 \pm 0,087 \\
0,437 \pm 0,023 \\
0,211 \pm 0,004 \\
0,000 \\
0,000
\end{gathered}
$$

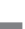


en nuestro país ya que la mayor parte de la leche recepcionada en planta viene de la Región de los Lagos y de los Ríos con un volumen superior al $71 \%$ del total de la leche nacional (25), lo cual indica que la leche fluida y la procesada tiene altos niveles de ALC, superior a la reportada por la literatura $(12,20,21)$.

Llama la atención que la leche descremada tenga alto ALC, lo cual, no concuerda con la idea generalizada de que el ALC está asociado con la grasa (26); para el caso de leche semidescremada se reporta valores de $0,01 \mathrm{~g} / 100 \mathrm{~g}$ (16); esto se podría explicar por efecto que tiene el procesamiento de la leche. Si bien la variación en la concentración de ALC en los productos lácteos es esencialmente una función de la concentración en la leche cruda, formada a través de las reacciones enzimáticas de los microorganismos del rumen, también se debería a futuras reacciones de isomerización durante la elaboración de los alimentos (11); reportándose que el procesamiento y almacenaje de productos a 60-80 ${ }^{\circ} \mathrm{C}$, afectaría la concentración de ALC y su estabilidad de (27) así como también lo afectaría la refrigeración (28).

En productos lácteos en Alemania, se reportan valores de $0.40 \mathrm{~g} / 100 \mathrm{~g}$ de ALC para quesos maduros $\mathrm{y}$ de 0,29 a $0,71 \mathrm{~g} / 100 \mathrm{~g}$ en grasa en quesos frescos (12) y de $0,39,0,18,0,16 \mathrm{~g} / 100 \mathrm{~g}$ para queso Beaufort, Blue y Camembert en Francia (20); habiéndose encontrado $0,48 \mathrm{~g} / 100 \mathrm{~g}$ en Portugal (21). Quesos como Parmesano y el Romano que tienen una edad de maduración mayor a 10 meses están entre los quesos con más baja cantidad de ALC, por otro lado quesos madurados con bacterias de envejecimiento de 4 a 8 semanas tienen alta cantidad de ALC. Otros datos referenciales en la cantidad de ALC para quesos muestran valores de $0,5 \mathrm{y}$ de $0,36 \mathrm{~g} / 100 \mathrm{~g}$ (29). En la mantequilla se reporta una cantidad de 0,48 $\mathrm{g} / 100 \mathrm{~g}(30)$ y $0,51 \mathrm{~g} / 100 \mathrm{~g}$ (20) y de $0,49 \mathrm{~g} / 100 \mathrm{~g}(21)$. Para leche condensada en nuestro trabajo se encontró niveles de 0,960 a 2,200 de ALC, muy superior a lo reportado en la literatura que no superan $\operatorname{los} 0,7 \mathrm{~g} / 100 \mathrm{~g}$ (29).

Esto indica la calidad de nuestros productos lácteos en ALC, ácido graso que ha demostrado ser un potente anticancerígeno en humanos, además tendría acción en el sistema inmune a través de dos mecanismos: por la habilidad del ALC de modificar la composición de la membrana celular (31) y por la habilidad de modular la actividad del receptor de reguladores de la proliferación peroxisomal (PPARs) (32). El ALC causaría una disminución en la masa de tejido adiposo, debido al efecto en el aumento al gasto energético (33), que puede ser secundario a una estimulación de actividad del sistema nervioso simpático (34). El ALC reduciría la respuesta de los lípidos al almacenaje en 3T3-L1 adipositos, inhibiendo la lipoproteina lipasa (35-36) y Stearoyl-CoA Desaturase-I (37).

\section{Isómeros del ALC en productos lácteos}

La tabla 2 muestra los isómeros de ALC. Podemos observar que el isómero cis-9, trans-11 se encuentra en un rango que va de 0,26 para el silo de leche en verano en los Ángeles y un máximo de 1,26 en mantequilla de la región de los Lagos. Este isómero porcentualmete representa en promedio el 53\% del total de ALC siendo más alto en la región de los Lagos. El cis-9, trans-11 se encuentra en mayor proporción según la literatura alcanzando un $75-90 \%$ del ALC total de grasa de la leche (5), siendo uno de los dos isómeros con acción biológica más conocida.

En el caso de la leche condensada este isómero varía de $0.43-0.73 \mathrm{~g} / 100 \mathrm{~g}$ dependiendo el año estudiado, reportándose en la literatura valores de $0,63 \mathrm{~g} / 100 \mathrm{~g}$ promedio (15) y de $0,70 \mathrm{~g} / 100 \mathrm{~g}$ (38). El isómero cis-9, trans-11 en el queso y mantequilla presentaron un rango de $0,37-1,47 \mathrm{~g} / 100 \mathrm{~g}$ y $1,22-2,22 \mathrm{~g} / 100 \mathrm{~g}$ respectivamente, siendo valores más altos a lo reportado en la literatura, que para el caso del queso alcanza rangos de 0,32 - 0,89 g/100g (39) y promedio de $0,35 \mathrm{~g} / 100 \mathrm{~g}$ (21). Para la mantequilla se observan valores de 0,94 para la mantequilla (15) y de $0,38 \mathrm{~g} / 100 \mathrm{~g}$ (21). Otros artículos señalan valores para la mantequilla de 1,32 $\mathrm{g} / 100 \mathrm{~g}$ indicándose que el procesamiento no afectó la relación de cis-9 trans-11 en ALC total (27); sin embargo, la pasteurización reduciría significativamente este isómero, incluso al calentarse la leche por cinco minutos con microondas (40).

El otro isómero encontrado en un rango de 0,152 a 0,430 en los silos de leche fue el trans-10, cis-12, pero no se encontró en las muestras de otoño y primavera. Este isómero está presente en cantidades de solo 3 a $5 \%$ del total de ALC a pesar de su importante rol fisiológico (41) y de menos del $1 \%$ en productos lácteos (21). Es por ello que cobra mucha importancia las proporciones encontradas en nuestros muestreos. A este isómero se le ha atribuido la capacidad de reducir la síntesis de grasa láctea (42). Además los ácidos grasos trans actuarían modificando el metabolismo de lípido en personas con sobrepeso las que al consumir 3.4 g ALC/día tendrían una reducción significativa de la masa de grasa corporal. En cerdos en crecimiento dar una mezcla de trans-10, cis-12 disminuye la grasa de cuerpo en los animales $(43,44)$. Además, los estudios sugieren que el trans-10, cis-12 pero no cis-9, trans-11 inhibe la proliferación y la diferenciación de 3T3-L1 preadiposito y las células cultivadas en el estroma vascular del tejido adiposo 
subcutáneo humano, diminuyendo la acumulación de lípidos (45). Evidencias indican que al aumentó la grasa intramuscular seria atribuible al reclutamiento de más células estromales vasculares por parte de ALC a los adipositos (46). Estos estudios sugirieren la regulación del ALC en el desarrollo del adiposito en tejido subcutáneo y en el intramuscular (47).

El isómero cis-10, cis-12 lo encontramos en alta proporción en silo de leche en primavera en la planta de Osorno y también se encontró en alta concentración en leche condensada y en leche en polvo. En relación a la cantidad total de ALC alcanzó sobre un 50\% en leche condensada, crema y leche en polvo; siendo el mayor isómero encontrado en Osorno en los silos de primavera. A este isómero no se le ha atribuido ningún efecto benéfico y en los estudios en leche muestra menos del 0.01 $\%$, no apareciendo en mantequilla, ni en quesos (21). Sin embargo, nosotros determinamos que el isómero cis-10, cis-12 se presentó en alta cantidad seguido del cis-9, trans-11, por lo cual cobra gran importancia determinar su rol biológico.

Para Alemania el consumo de ALC provenientes de productos lácteos es de 0,24 g/día (12), en Francia de 0,17-0,21 g/día (20) y en Portugal de 0,40-0,722 g/ día, sugiriéndose que consumos medios óptimos para humanos es de 15-20 g/día (49) y consumo ricos en ALC de $65 \mathrm{~g} / \mathrm{dia}$ (50) total en los alimentos. Ip y col. (51) estimó que una persona de $70 \mathrm{~kg}$ que consume 3.0 $\mathrm{g}$ de ALC/día obtiene un máximo beneficio en su salud. De acuerdo a los valores de ALC encontrados y considerando que el consumo de leche en Chile es de $127 \mathrm{I} /$ persona (25) determinamos un consumo diario de ALC equivalente a $0.03 \mathrm{~g} /$ día - 0,24 g/día, esto tomando los valores extremos de ALC obtenidos en muestro estudio. Por lo que concluimos que, si bien el consumo de productos lácteos es más bajo que los países desarrollados, la alta cantidad de ALC de nuestra leche nos garantiza óptimos niveles para la salud humana.

\section{RESUMEN}

Se midieron las cantidades de acido linoleico conjugado (ALC) - isómeros: cis-9, trans-11, trans-10, cis-12 y cis-10, cis-12 -en leche de estanque de la empresa NESTLE y de alimentos lácteos en Chile, mediante cromatografía de gas. El ALC encontrado fue más alto $(p<0.05)$ en las muestras tomadas en la ciudad de Osorno comparado con la leche de la ciudad de Los Angeles. A nivel estacional el valor más alto de ALC de leche líquida fue encontrado durante la primavera en la ciudad Osorno $(1,72 \mathrm{~g} / 100 \mathrm{~g})$, mientras que el valor más bajo se presentó en invierno $(0,415 \mathrm{~g} / 100 \mathrm{~g})$ en la ciudad de Los Ángeles. La cantidad promedio de ALC en leche en polvo y de leche condensada fue de 1,967 y $1,493 \mathrm{~g} / 100 \mathrm{~g}$ respectivamente durante el año 2004. La mantequilla, el queso y la crema presentaron promedios de $1,502,0,883$ y $1,900 \mathrm{~g} / 100 \mathrm{~g}$ respectivamente de ALC. Se concluye que los productos lácteos analizados tienen altos valores de ALC en Chile.

Palabras clave: ALC; ácido linoleico conjugado; leche; productos lácteos.

Dirigir la correspondencia a:

Profesor

Juan Pablo Avilez R.

Escuela de Medicina Veterinaria

Facultad de Recursos Naturales

Universidad Católica de Temuco

Montt 56 - Temuco

Teléfono: 45-205554-205550

Fax: 45-205570

E-mail:.jpavilez@uct.cl

Agradecimientos: Proyecto FONDEF N ${ }^{\circ}$ D02I1135. "Desarrollo de productos con alto contenido de principios activos funcionales a partir de leche bovina y sus derivados".

\section{BIBLIOGRAFÍA}

1. Pariza MW, Park ME. The biologically active isomers of conjugated linoleic acid. Prog Lipid Res 2001; 40: 283-298.

2. O`Quinn PR, Nelssen JL, Goodband RD, Tokach MD. Conjugated linoleic acid. Anim Health Res Rev 2000; 1:35-46.

3. Kay JK, Mackle TR, Auldist MJ, Thomson NA, Bauman DE. "Endogenous synthesis and enhancement of conjugated linoleic acid in pasture-fed dairy cows". Proc New Zealand Soc Animal Product 2002; 62:12-15.

4. Ward AT, Wittenberg KM, Froebe HM, Przybylski R, Malcolmson L. Fresh Forage and Solin Supplementation on Conjugated Linoleic Acid Levels in Plasma and Milk. J Dairy Sci 2003; 86:17421750.

5. Ip C, Banni S, Angioni E, Carta G, McGinley J, Thompson HJ, Barbano D, Bauman D. Conjugated linoleic acidenriched butter fat alters mammary gland morphogenesis and reduces cancer risk in rats. J Nutr 1999; 129:2135-2142.

6. Blankson H, Stakkestad JA, Erling HF, Wadstein TJ, Gudmundsen O. Conjugated linoleic acid reduces body fat mass in overweigth and obese humans. $\mathrm{J}$ Nutr 2000; 130: 2943-2948.

7. Lee KN, Kritchevsky D, Pariza MW. Conjugated 
linoleic acid and atherosclerosis in rabbits. Atherosclerosis 1994; 108:19-25.

8. Houseknecht KL, Vanden Heuvel JP, MoyaCamarena SY, Portocarrero CP, Peck LW, Nickel KP, Belury MA. Dietary conjugated linoleic acid normalizes impaired glucose tolerance in the Zucker diabetic fatty fa/fa rat. Biochem Biophys Res Comm 1998; 244:678-682.

9. Sugano M, Tsujita A, Yamasaki M, Noguchi M, Yamada K. Conjugated linoleic acid modulates tissue levels of chemical mediator and inmune globulins in rats. Lipids. 1998; 33: 521-527.

10. Yu L. Free radical scavenging properties of conjugated linoleic acid. J Agric Food Chem 2001; 49 3452-3456.

11. Lin HD, Boylston MJ, Chang LO, Luedecke H, Shultz TD. Survey of the Conjugated Linoleic Acid Contents of Dairy Products. Dairy Science 1995; 78 (11) 2358-2365.

12. Fritsche J, Steinhart H. Amounts of conjugated linoleic acid (CLA) in German foods evaluation of daily intake. Z. Lebensm Unters Forsh A 1998; 206: 77-82.

13. Bauman DE, Griinari JM. Regulation and nutritional manipulation of milk fat: Low-fat milk syndrome. Livest Prod Sci 2001; 70: 15-29.

14. Dhiman TR, Helmink ED, McMahon DJ, Fife RL, Pariza MW. Conjugated linoleic acid content of milk and cheese from cows fed extruded oilseeds. J Dairy Sci 1999; 82:412-419.

15. Gagliostro GA, Vidaurreta LI, Schroeder GF, Rodriguez A, Gatti P. Incrementando los valores basales de ácido linoleico conjugado (ALC) en la grasa butirosa de vacas lecheras en condiciones de pastoreo. Rev Arg Prod Anim 22 (Suplem 1) 2002; 59-60.

16. Pinto M, Rubilar C, Carrasco E. Efecto estacional y del área geográfica en la composición de ácidos grasos en la leche de bovinos. Agro sur 2002; 30 (2): 75-90.

17. International Dairy Federation. Milk and milk products-Guidance on sampling. FIL-IDF Standard 50 C. Int. Dairy Fed., Brussels, Belgium. 1995.

18. Folch J, Less H, Sloane-Stanley GH. A simple method for the insolation and purification of total lipids from animal tissue. J Biol Chem 1957; 726: 497-509.

19. Morrison WR, Smith LM. Preparation of fatty acids methyl esters and dimethylacetals from lipids with boron fluoride-methanol. J Lip Res 1964; 5:600-608.

20. Laloux L, du Chaffaut L, Razanamahefa L, Lafay
L. Trans fatty acid content of foods and intake levels in France. Eur J Lipid Sci Technol 2007; 109: 918-929

21. Susana V. Martins SV, Lopes PA, Alfaia CM, Ribeiro VS, Guerreiro TV, Fontes CM, Castro MF, Soveral G, Prates JA. Contents of conjugated linoleic acid isomers in ruminant-derived foods and estimation of their contribution to daily intake in Portugal. British J Nutr 2007; 98: 1206-1213.

22. Balocchi O, Pinochet D, Wittwer F, Contreras PA, Echeverría R, Guzmán F. Rendimiento y composición mineral del forraje de una pradera permanente fertilizada con magnesio. Pesq Agropecurias 2001; 36, 1309-1317

23. Thomson NA, Chand A, Kay JK. Predicting $\Delta 9$-desaturase activity and the association with conjugate linoleic acid (ALC) concentration in bovine milk. Proc New Zeland Soc Animal Product 2003; 63: 25-30.

24. Khanal R, Olson KC. Factors Affecting Conjugated Linoleic Acid (ALC) Content in Milk, Meat, and Egg: A Review . Department of Animal, Dairy and Veterinary Sciences, Asian Network for Scientific Information. Utah State University, Logan, UT 84322, USA. Pakistan J Nutr 2004;3(2):82-98.

25. Ministerio de Agricultura. Oficina de Estudios y Políticas Agrarias (ODEPA). Estadísticas agropecuarias. Santiago: ODEPA; 2007. Disponible en URL: http://www.odepa.gob.cl

26. Bauma N, Barbano DM, Dwyer DA, Griinari IM. Technical note: Production of butter with enhanced conjugated linoleic acid for use in biomedical studies with animal models. J Dairy Sci 2000; 83: 2422-2425.

27. Shantha NC, Ram LN, O'Leary J, Hicks CL, Decker EA. Conjugated linoleic acid concentrations in dairy products as affected by processing and storage. $\mathrm{J}$ Food Sci 1995; 60:695-697.

28. Rodríguez-Alcala LM, Fontechal J. Hot Topic: Fatty Acid and Conjugated Linoleic Acid (CLA) Isomer Composition of Commercial CLA-Fortified Dairy Products: Evaluation After Processing and Storage. J Dairy Sci 2007; 90: (5) 2083-2090

29. Chin SF, Liu W, Storkson JM, Ha YL, Pariza MW. Dietary sources of conjugated dienoic isomers of linoleic acid, a newly recognized alcss of anticarcinogens. J Food Comp Anal 1992; 5:185-197.

30. Mir PS, Okine EK, Goonewardene L, He ML, Mir Z. Effects of synthetic conjugated linoleic acid (CLA) or bio-formed CLA as high CLA beef on rat growth and adipose tissue development. Canadian J Animal Sci $2003 ; 83(3): 583-592$. 
31. Pariza MW, Park Y, Cook ME. Mechanisms of actionof conjugated linoleic acid: evidence and speculation. Proc Soc Exp Biol Med 2000; 223: 8-13.

32. Bassaganya-Riera J, Hontecillas R, Beitz D. Colonic anti-inflammatory mechanisms of conjugated linoleic acid. Clin Nutr 2002; 21: 451-459

33. West DB, Delany JP, Camet PM, Blohm F, Truett AA, Scimeca J. Effects of conjugated linoleic acid on body fat and energy metabolism in the mouse. Am J Physiol 1998; 275: R667- R672.

34. Ohnuki K, Haramizu S, Oki K, Ishihara K, Fushiki T. A single oral administration of conjugated linoleic acid enhanced energy metabolism in mice. Lipids 2001; 36: 583-587.

35. Park Y, Albright KJ, Storkson JM, Liu W, Cook ME, Pariza MW. Changes in body composition in mice during feeding and withdrawal of conjugated linoleic acid. Lipids 1999; 34: 243-248.

36. Lin Y, Schuurbiers E, Van der Veen S, De Deckere EA. Conjugated linoleic acid isomers have differential effects on triglyceride secretion in Hep G2 cells. Biochim Biophys Acta 2001;1533: 38-46.

37. Choi Y, Park Y, Pariza MW, Ntambi JM. Regulationof stearoyl-CoA desaturase activity by the trans-10,cis-12 isomer of conjugated linoleic acid in HepG2 cells. Biochem Biophys Res 2001; 284: 689-693.

38. Shantha NC, Crum AD, Decker EA. Evaluation of conjugated linoleic acid concentrations in cooked beef. J Agricul Food Chem 1994; 42 (8)17571760.

39. Campbell W, Drake MA, Larick DK. The impact of fortification with conjugated linoleic acid (ALC) on the quality of fluid milk. J Dairy Sci 2003; 86: 43-51.

40. Herzallah SM, Humeid MA, Al-Ismail KM. Effect of Heating and Processing Methods of Milk and Dairy Products on Conjugated Linoleic Acid and Trans Fatty Acid Isomer Content Dairy Sci 2005; 88:1301-1310.

41. Parodi PW. Conjugate linoleic acid and other anticarcinogenic agents of bovine milk fat. J Dairy Sci 1999; 82:1339-1349.

42. Perfield JWII, Lock AL, Griinari JM, Sæbo A, Delmonte P, Dwyer DA, Barman DE. trans-9, cis-
11 Conjugated Linoleic Acid Reduces Milk Fat Synthesis in Lactating Dairy Cows. J Dairy Sci 2007; 90:2211-2218.

43. Baumgard LH, Matitashvili BA, Corl DA, Dwyer D, Bauman DE. "trans-10, cis-12 Conjugated linoleic acid decreases lipogenic rates and expression of genes involved in milk lipid synthesis in dairy cows". J Dairy Sci 2002; 85: 2155-2163.

44. Dugan ME, Aalhus JL, Kramer JK. Conjugated linoleic acid pork research. Am J Clin Nutr 2004 (79) (Suppl.) 1212-1216.

45. Brown JM, Boysen MS, Jensen SS, Morrison RF, Storkson J, Currie RL, Pariza M, Mandrup S, McIntosh MK. Isomer-specific regulation of metabolism and PPARg signaling by CLA in human preadipocytes. J Lipid Res 2003;44:1287-1300.

46. Meadus WJ, MacInnis R, Dugan ME. Prolonged dietary treatment with conjugated linoleic acid stimulates porcine muscle peroxisome proliferator activated receptor $\mathrm{g}$ and glutaminefructose aminotransferase gene expression in vivo. J Mol Endocrinol 2002;28: 79-86.

47. Hausman GJ, Poulos S. Recruitment and differentiation of IM preadipocytes in stromal-vascular cell cultures derived from neonatal pig semitendinosus muscles. J Anim Sci 2004; 82: 429-437.

48. Wallace RJ, McKain N, Kevin J, Shingfield G, Devillard E. Isomers of conjugated linoleic acids are synthesized via different mechanisms in ruminal digesta and bacteria. J Lipid Res 2007;48:22472254.

49. Ritzenthaler KL, McGuire MK, Falen R, Shultz TD, Dasgupta N, McGuire MA. Estimation of conjugated linoleic acid intake by written dietary assessment methodologies underestimates actual intake evaluated by food duplicate methodology. J Nutr 2001; 131: 1548-1554.

50. Park Y, McGuire, MK, Behr R, McGuire MA, Evans MA, Shultz TD. High-fat dairy product consumption increases delta 9c,11t-18:2 (rumenic acid) and total lipid concentrations of human milk. Lipids 1999;34:543-549.

51. Ip C, Thompson HJ, Scimeca JA. Conjugated linoleic acid suppresses mammary carcinogenesis and proliferative activity of the mammary gland in the rat. Cancer Res 1994;54:1212-1215. 